\title{
Integrable equations on time scales
}

Metin Gürses, Gusein Sh. Guseinov, and Burcu Silindir

Citation: Journal of Mathematical Physics 46, 113510 (2005); doi: 10.1063/1.2116380

View online: http://dx.doi.org/10.1063/1.2116380

View Table of Contents: http://aip.scitation.org/toc/jmp/46/11

Published by the American Institute of Physics

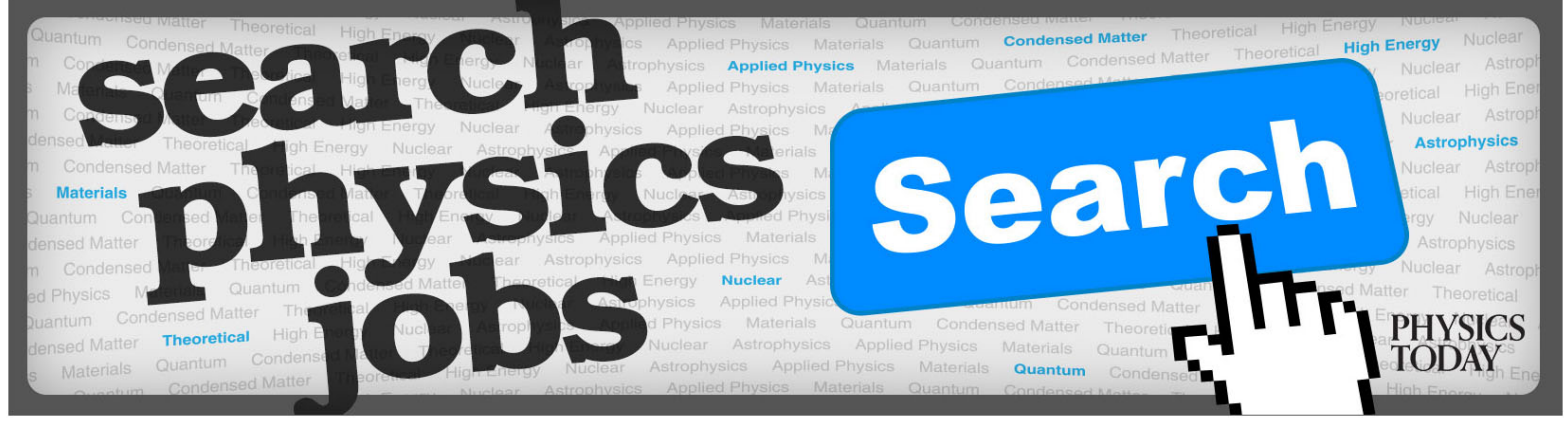




\title{
Integrable equations on time scales
}

\author{
Metin Gürses \\ Department of Mathematics, Faculty of Sciences, Bilkent University, \\ 06800 Ankara, Turkey \\ Gusein Sh. Guseinov \\ Department of Mathematics, Atilim University, 06836 Incek, Ankara, Turkey \\ Burcu Silindir \\ Department of Mathematics, Faculty of Sciences, Bilkent University, \\ 06800 Ankara, Turkey
}

(Received 14 June 2005; accepted 19 September 2005; published online 15 November 2005)

\begin{abstract}
Integrable systems are usually given in terms of functions of continuous variables (on R), in terms of functions of discrete variables (on Z), and recently in terms of functions of $q$-variables (on $\mathbb{K}_{q}$ ). We formulate the Gel'fand-Dikii (GD) formalism on time scales by using the delta differentiation operator and find more general integrable nonlinear evolutionary equations. In particular they yield integrable equations over integers (difference equations) and over $q$-numbers ( $q$-difference equations). We formulate the GD formalism also in terms of shift operators for all regular-discrete time scales. We give a method allowing to construct the recursion operators for integrable systems on time scales. Finally, we give a trace formula on time scales and then construct infinitely many conserved quantities (Casimirs) of the integrable systems on time scales. (C) 2005 American Institute of Physics.
\end{abstract}

[DOI: $10.1063 / 1.2116380]$

\section{INTRODUCTION}

Integrable systems are well studied and well understood in $1+1$ dimensions. ${ }^{1-3}$ Here one of the dimensions denotes the time (evolution) variable and the other one denotes the space variable which is usually taken as continuous. There are also important examples where this variable takes values in Z, i.e., integer values. In both cases the Gel'fand-Dikii (GD) approach is quite effective. One can generate hierarchies of integrable evolution equations, both on $\mathbb{R}$ and on $\mathbb{Z}$ (see Ref. 3 for GD applications and related references). In addition one can construct the conserved quantities, Hamilton operators, and recursion operators. Investigation of integrable systems on $q$-discrete intervals started in Refs. 4-6. They considered GD formalism on $\mathbb{K}_{q}$ and found $q$-integrable hierarchies including the $q$-KdV equation.

In this work we extend the Gel'fand-Dikii approach to time scales where $\mathbb{R}, \mathbb{Z}$, and $\mathbb{K}_{q}$ are special cases. In the next section we give a brief review of time scales calculus. See Refs. 7-13 for a more detailed review of the subject. In GD formalism, in obtaining integrable systems the essential tools are the differential and shift operators and their inverses. For extending the GD formulation to time scales we give the necessary means to construct in the sequel the algebra of pseudo- $\Delta$-differential operators and the algebra of shift operators. In Sec. III we assume $\Delta$-differential Lax operators and derive the $\Delta$-Burgers hierarchy with its recursion operator. We present special cases of the Burgers equation for $\mathbb{T}=h Z$ and $\mathbb{T}=\mathbb{K}_{q}$. In Sec. IV, we consider the regular time scales where the inverse of jump operators can be defined. Here we assume a pseudo$\Delta$-differential algebra and give the corresponding GD formulation. As an example we present a $\Delta$-KdV hierarchy. We first find $n=1$ member of the hierarchy and write out it explicitly for $\mathbb{T}$ $=\mathrm{R}, \mathrm{Z}, \mathbb{K}_{q}$ and for $\mathbb{T}=(-\infty, 0) \cup \mathbb{K}_{q}$. Then we give the $n=3$ member and call it as the $\Delta-\mathrm{KdV}$ system. We call it $\Delta$-KdV equation, because the corresponding Lax operator is a second order $\Delta$-differential operator. It involves two fields $u$ and $v$, but the second field $v$ can be expressed in 
terms of the first filed $u$. When $\mathbb{T}=\mathbb{R}$, this system reduces to the standard KdV equation. In Sec. V, we consider the regular-discrete time scales and introduce the algebra of shift operators on them and give the corresponding GD formulation for all such time scales. Here several examples are presented. We first generalize the examples of discrete systems on Z given in Ref. 3 (one field, two fields, and four fields examples in Ref. 3) to arbitrary discrete time scales. In all these examples when $\mathbb{T}=\mathbb{Z}$ we get the discrete evolutions given in Ref. 3 . We construct the recursion operators of these systems on time scales. We generalize the Frenkel's $\mathrm{KdV}$ system ${ }^{4}$ introduced on $\mathbb{K}_{q}$ to arbitrary discrete time scales and we construct its recursion operator. In this section, we finally give an example of the KP hierarchy on discrete time scales. In Sec. VI, we extend the standard way of constructing the conserved quantities of integrable systems to time scales by introducing a trace form on the algebra of $\Delta$-pseudo-differential operators. The trace form introduced in this section reduces, in particular cases, to the standard trace forms on $\mathbb{R}$ and Z. In the Appendix we give the recursion operators of two four-fields systems introduced in Sec. V. We end up with a conclusion.

\section{TIME SCALE CALCULUS}

The time scale calculus is developed mainly to unify differential, difference, and $q$-calculus. A time scale (T) is an arbitrary nonempty closed subset of the real numbers. The calculus of time scales was initiated by Aulbach and Hilger, ${ }^{7,8}$ in order to create a theory that can unify and extend discrete and continuous analysis. The real numbers $(\mathbb{R})$, the integers $(\mathbb{Z})$, the natural numbers $(\mathbb{N})$, the non-negative integers $\left(\mathbb{N}_{0}\right)$, the $h$-numbers $(h \mathbb{Z}=\{h k: k \in \mathbb{Z}\}$, where $h>0$ is a fixed real number), and the $q$-numbers $\left(\mathbb{K}_{q}=q^{\mathbb{Z}} \cup\{0\} \equiv\left\{q^{k}: k \in \mathbb{Z}\right\} \cup\{0\}\right.$, where $q>1$ is a fixed real number) are examples of time scales, as are $[0,1] \cup[2,3],[0,1] \cup \mathbb{N}$, and the Cantor set, where $[0,1]$ and $[2,3]$ are real number intervals. In Refs. 7 and 8 Aulbach and Hilger introduced also dynamic equations on time scales in order to unify and extend the theory of ordinary differential equations, difference equations, and quantum equations ${ }^{9}$ ( $h$-difference and $q$-difference equations based on $h$-calculus and $q$-calculus, respectively). For a general introduction to the calculus on time scales we refer the reader to the textbooks by Bohner and Peterson. ${ }^{10,11}$ Here we give only those notions and facts connected to time scales which we need for our purpose in this paper.

Any time scale $\mathrm{T}$ is a complete metric space with the metric (distance) $d(x, y)=|x-y|$ for $x, y \in \mathbb{T}$. Consequently, according to the well-known theory of general metric spaces, we have for $\mathrm{T}$ the fundamental concepts such as open balls (intervals), neighborhood of points, open sets, closed sets, compact sets, and so on. In particular, for a given number $r>0$, the $r$-neighborhood $U_{r}(x)$ of a given point $x \in \mathbb{T}$ is the set of all points $y \in \mathbb{T}$ such that $d(x, y)<r$. By a neighborhood of a point $x \in \mathbb{T}$ is meant an arbitrary set in $\mathbb{T}$ containing an $r$-neighborhood of the point $x$. Also we have for functions $f: T \rightarrow \mathbb{R}$ the concepts of the limit, continuity, and properties of continuous functions on general complete metric spaces (note that, in particular, any function $f: Z \rightarrow \mathbb{R}$ is continuous at each point of $\mathbb{Z}$ ). The main task is to introduce and investigate the concept of derivative for functions $f: T \rightarrow R$. This proves to be possible due to the special structure of the metric space T. In the definition of derivative, the so-called forward and backward jump operators play special and important roles.

Definition 1: For $x \in \mathbb{T}$ we define the forward jump operator $\sigma: \mathbb{T} \rightarrow \mathbb{T}$ by

$$
\sigma(x)=\inf \{y \in \mathbb{T}: y>x\},
$$

while the backward jump operator $\rho: \mathrm{T} \rightarrow \mathrm{T}$ is defined by

$$
\rho(x)=\sup \{y \in \mathbb{T}: y<x\} .
$$

In this definition we set in addition $\sigma(\max \mathbb{T})=\max \mathbb{T}$ if there exists a finite $\max \mathbb{T}$, and $\rho(\min \mathbb{T})=\min \mathbb{T}$ if there exists a finite min $\mathbb{T}$. Obviously both $\sigma(x)$ and $\rho(x)$ are in $\mathbb{T}$ when $x$ $\in \mathbb{T}$. This is because of our assumption that $\mathbb{T}$ is a closed subset of $\mathbb{R}$.

Let $x \in \mathbb{T}$. If $\sigma(x)>x$, we say that $x$ is right-scattered, while if $\rho(x)<x$ we say that $x$ is left-scattered. Also, if $x<\max \mathbb{T}$ and $\sigma(x)=x$, then $x$ is called right-dense, and if $x>\min \mathbb{T}$ and 
$\rho(x)=x$, then $x$ is called left-dense. Points that are right-scattered and left-scattered at the same time are called isolated. Finally, the graininess functions $\mu, \nu: T \rightarrow[0, \infty)$ are defined by

$$
\mu(x)=\sigma(x)-x, \quad \text { and } \nu(x)=x-\rho(x) \quad \text { for all } x \in \mathbb{T} .
$$

Example 1: If $\mathbb{T}=\mathbb{R}$, then $\sigma(x)=\rho(x)=x$ and $\mu(x)=\nu(x)=0$. If $\mathbb{T}=h \mathbb{Z}$, then $\sigma(x)=x+h, \rho(x)$ $=x-h$, and $\mu(x)=\nu(x)=h$. On the other hand, if $\mathbb{T}=\mathbb{K}_{q}$ then we have

$$
\sigma(x)=q x, \quad \rho(x)=q^{-1} x, \quad \mu(x)=(q-1) x, \quad \text { and } \nu(x)=\left(1-q^{-1}\right) x .
$$

Let $\mathbb{T}^{\kappa}$ denote Hilger's above truncated set consisting of T except for a possible left-scattered maximal point. Similarly, $\mathbb{T}_{\kappa}$ denotes the below truncated set obtained from $\mathbb{T}$ by deleting a possible right-scattered minimal point.

Definition 2: Let $f: T \rightarrow R$ be a function and $x \in \mathbb{T}^{\kappa}$. Then the delta derivative of $f$ at the point $x$ is defined to be the number $f^{\Delta}(x)$ (provided it exists) with the property that for each $\varepsilon>0$ there exists a neighborhood $U$ of $x$ in $T$ such that

$$
\left|f(\sigma(x))-f(y)-f^{\Delta}(x)[\sigma(x)-y]\right| \leqslant \varepsilon|\sigma(x)-y|,
$$

for all $y \in U$.

Remark 1: If $x \in \mathbb{T} \backslash \mathbb{T}^{\kappa}$, then $f^{\Delta}(x)$ is not uniquely defined, since for such a point $x$, small neighborhoods $U$ of $x$ consist only of $x$ and besides we have $\sigma(x)=x$. Therefore (5) holds for an arbitrary number $f^{\Delta}(x)$. This is a reason why we omit a maximal left-scattered point.

We have the following: (i) If $f$ is delta differentiable at $x$, then $f$ is continuous at $x$. (ii) If $f$ is continuous at $x$ and $x$ is right-scattered, then $f$ is delta differentiable at $x$ with

$$
f^{\Delta}(x)=\frac{f(\sigma(x))-f(x)}{\mu(x)} .
$$

(iii) If $x$ is right-dense, then $f$ is delta differentiable at $x$ iff the limit

$$
\lim _{y \rightarrow x} \frac{f(x)-f(y)}{x-y}
$$

exists as a finite number. In this case $f^{\Delta}(x)$ is equal to this limit. (iv) If $f$ is delta differentiable at $x$, then

$$
f(\sigma(x))=f(x)+\mu(x) f^{\Delta}(x) .
$$

Definition 3: If $x \in \mathbb{T}_{\kappa}$, then we define the nabla derivative of $f: T \rightarrow R$ at $x$ to be the number $f^{\nabla}(x)$ (provided it exists) with the property that for each $\varepsilon>0$ there is a neighborhood $U$ of $x$ in $T$ such that

$$
\left|f(\rho(x))-f(y)-f^{\nabla}(x)[\rho(x)-y]\right| \leqslant \varepsilon|\rho(x)-y|,
$$

for all $y \in U$.

We have the following: (i) If $f$ is nabla differentiable at $x$, then $f$ is continuous at $x$. (ii) If $f$ is continuous at $x$ and $x$ is left-scattered, then $f$ is nabla differentiable at $x$ with

$$
f^{\nabla}(x)=\frac{f(x)-f(\rho(x))}{\nu(x)} .
$$

(iii) If $x$ is left-dense, then $f$ is nabla differentiable at $x$ if and only if the limit 


$$
\lim _{y \rightarrow x} \frac{f(x)-f(y)}{x-y}
$$

exists as a finite number. In this case $f^{\nabla}(x)$ is equal to this limit. (iv) If $f$ is nabla differentiable at $x$, then

$$
f(\rho(x))=f(x)-\nu(x) f^{\nabla}(x) .
$$

Example 2: If $\mathbb{T}=\mathrm{R}$, then $f^{\Delta}(x)=f^{\nabla}(x)=f^{\prime}(x)$, the ordinary derivative of $f$ at $x$. If $\mathbb{T}=h Z$, then

$$
f^{\Delta}(x)=\frac{f(x+h)-f(x)}{h} \quad \text { and } f^{\nabla}(x)=\frac{f(x)-f(x-h)}{h} .
$$

If $\mathbb{T}=\mathbb{K}_{q}$, then

$$
f^{\Delta}(x)=\frac{f(q x)-f(x)}{(q-1) x} \text { and } f^{\nabla}(x)=\frac{f(x)-f\left(q^{-1} x\right)}{\left(1-q^{-1}\right) x},
$$

for all $x \neq 0$, and

$$
f^{\Delta}(0)=f^{\nabla}(0)=\lim _{y \rightarrow 0} \frac{f(y)-f(0)}{y}
$$

provided that this limit exists.

Among the important properties of the delta differentiation on $\mathbb{T}$ we have the Leibnitz rule, if $f, g: T \rightarrow \mathbb{R}$ are delta differentiable functions at $x \in \mathbb{T}^{\kappa}$, then so is their product $f g$ and

$$
\begin{gathered}
(f g)^{\Delta}(x)=f^{\Delta}(x) g(x)+f\left(\sigma(x) g^{\Delta}(x)\right. \\
\quad f(x) g^{\Delta}(x)+f^{\Delta}(x) g(\sigma(x)) .
\end{gathered}
$$

Also, if $f, g: T \rightarrow R$ are nabla differentiable functions at $x \in \mathbb{T}_{\kappa}$, then so is their product $f g$ and

$$
\begin{gathered}
(f g)^{\nabla}(x)=f^{\nabla}(x) g(x)+f\left(\rho(x) g^{\nabla}(x),\right. \\
=f(x) g^{\nabla}(x)+f^{\nabla}(x) g(\rho(x)) .
\end{gathered}
$$

In the next proposition we give a relationship between the delta and nabla derivatives (see Ref. 12).

Proposition 4: (i) Assume that $f: \mathbb{T} \rightarrow \mathbb{R}$ is delta differentiable on $\mathbb{T}^{\kappa}$. Then $f$ is nabla differentiable at $x$ and

$$
f^{\nabla}(x)=f^{\Delta}(\rho(x)),
$$

for $x \in \mathbb{T}_{\kappa}$ such that $\sigma(\rho(x))=x$. If, in addition, $f^{\Delta}$ is continuous on $\mathbb{T}^{\kappa}$, then $f$ is nabla differentiable at $x$ and (20) holds for any $x \in \mathbb{T}_{\kappa}$.

(ii) Assume that $f: \mathbb{T} \rightarrow \mathbb{R}$ is nabla differentiable on $\mathbb{T}_{\kappa}$. Then $f$ is delta differentiable at $x$ and

$$
f^{\Delta}(x)=f^{\nabla}(\sigma(x)),
$$

for $x \in \mathbb{T}^{\kappa}$ such that $\rho(\sigma(x))=x$. If, in addition, $f^{\nabla}$ is continuous on $\mathbb{T}_{\kappa}$, then $f$ is delta differentiable at $x$ and (21) holds for any $x \in \mathbb{T}^{\kappa}$.

Now we introduce the concept of integral for functions $f: T \rightarrow R$.

Definition 5: A function $F: \mathbb{T} \rightarrow \mathbb{R}$ is called a $\Delta$-antiderivative of $f: \mathbb{T} \rightarrow \mathbb{R}$ provided $F^{\Delta}(x)$ $=f(x)$ holds for all $x$ in $T^{\kappa}$. Then we define the $\Delta$-integral from a to $b$ of $f$ by 


$$
\int_{a}^{b} f(x) \Delta x=F(b)-F(a) \quad \text { for all } a, b \in \mathrm{T} .
$$

Definition 6: A function $\Phi: \mathbb{T} \rightarrow \mathbb{R}$ is called a $\nabla$-antiderivative of $f: \mathbb{T} \rightarrow \mathbb{R}$ provided $\Phi^{\nabla}(x)$ $=f(x)$ holds for all $x$ in $\mathrm{T}_{\kappa}$. Then we define the $\nabla$-integral from $a$ to $b$ of $f$ by

$$
\int_{a}^{b} f(x) \nabla x=\Phi(b)-\Phi(a) \quad \text { for all } a, b \in \mathbb{T} .
$$

If $a, b \in \mathbb{T}$ with $a \leqslant b$ we define the closed interval $[a, b]$ in $\mathbb{T}$ by

$$
[a, b]=\{x \in \mathbb{T}: a \leqslant x \leqslant b\} .
$$

Open and half-open intervals, etc., are defined accordingly. Below all our intervals will be time scale intervals

Example 3: Let $a, b \in \mathbb{T}$ with $a<b$. Then we have the following.

(i) If $f: T=\mathbb{R}$ then

$$
\int_{a}^{b} f(x) \Delta x=\int_{a}^{b} f(x) \nabla x=\int_{a}^{b} f(x) \mathrm{d} x,
$$

where the integral on the right-hand side is the ordinary integral.

(ii) If $[a, b]$ consists of only isolated points, then

$$
\int_{a}^{b} f(x) \Delta x=\sum_{x \in[a, b)} \mu(x) f(x) \quad \text { and } \int_{a}^{b} f(x) \nabla x=\sum_{x \in(a, b]} \nu(x) f(x) .
$$

In particular, if $T=\mathbb{Z}$, then

$$
\int_{a}^{b} f(x) \Delta x=\sum_{k=a}^{b-1} f(k) \quad \text { and } \int_{a}^{b} f(x) \nabla x=\sum_{k=a+1}^{b} f(k) .
$$

If $T=h Z$, then

$$
\int_{a}^{b} f(x) \Delta x=h \sum_{x \in[a, b)} f(x) \quad \text { and } \int_{a}^{b} f(x) \nabla x=h \sum_{x \in(a, b]}^{b} f(x)
$$

and if $\mathrm{T}=\mathrm{K}_{q}$, then

$$
\int_{a}^{b} f(x) \Delta x=(1-q) \sum_{x \in[a, b)} x f(x) \text { and } \int_{a}^{b} f(x) \nabla x=\left(1-q^{-1}\right) \sum_{x \in(a, b]} x f(x) .
$$

The following relationship between the delta and nabla integrals follows from Definitions 5 and 6 by using Proposition 4 .

Proposition 7: If the function $f: \mathrm{T} \rightarrow \mathrm{R}$ is continuous, then for all $a, b \in \mathrm{T}$ with $a<b$ we have

$$
\int_{a}^{b} f(x) \Delta x=\int_{a}^{b} f(\rho(x)) \nabla x \text { and } \int_{a}^{b} f(x) \nabla x=\int_{a}^{b} f(\sigma(x)) \Delta x .
$$

Indeed, if $F: \mathbb{T} \rightarrow \mathbb{R}$ is a $\Delta$-antiderivative for $f$, then $F^{\Delta}(x)=f(x)$ for all $x \in \mathbb{T}^{\kappa}$, and by Proposition 4 we have $f(\rho(x))=F^{\Delta}(\rho(x))=F^{\nabla}(x)$ for all $x \in \mathbb{T}_{\kappa}$, so that $F$ is a $\nabla$-antiderivative for $f(\rho(x))$. Therefore 


$$
\int_{a}^{b} f(\rho(x)) \nabla x=F(b)-F(a)=\int_{a}^{b} f(x) \Delta x .
$$

From (16)-(21) and (30) we have the following integration by parts formulas: If the functions $f, g: \mathbb{T} \rightarrow \mathrm{R}$ are delta and nabla differentiable with continuous derivatives, then

$$
\begin{gathered}
\int_{a}^{b} f^{\Delta}(x) g(x) \Delta x=\left.f(x) g(x)\right|_{a} ^{b}-\int_{a}^{b} f(\sigma(x)) g^{\Delta}(x) \Delta x, \\
\int_{a}^{b} f^{\nabla}(x) g(x) \nabla x=\left.f(x) g(x)\right|_{a} ^{b}-\int_{a}^{b} f(\rho(x)) g^{\nabla}(x) \nabla x, \\
\int_{a}^{b} f^{\Delta}(x) g(x) \Delta x=\left.f(x) g(x)\right|_{a} ^{b}-\int_{a}^{b} f(x) g^{\nabla}(x) \nabla x, \\
\int_{a}^{b} f^{\nabla}(x) g(x) \nabla x=\left.f(x) g(x)\right|_{a} ^{b}-\int_{a}^{b} f(x) g^{\Delta}(x) \Delta x .
\end{gathered}
$$

For more general treatment of the delta integral on time scales (Riemann and Lebesgue delta integrals on time scales) see Ref. 13 and Chap. 5 of Ref. 11.

\section{BURGERS EQUATION ON TIME SCALES}

The Gel'fand-Dikii approach is very effective in studying the symmetries, bi-Hamiltonian formulation, and in constructing the recursion operators of integrable nonlinear partial differential equations. In this approach one takes the Lax operator $L$ in an algebra like a differential or pseudodifferential algebra, a matrix algebra, a polynomial algebra, or the Moyal algebra. In this section we take $L$ in the algebra of delta-differential operators.

Let $\mathrm{T}$ be a time scale. We say that a function $f: T \rightarrow R$ is $\Delta$-smooth if it is infinitely $\Delta$-differentiable (and hence infinitely $\nabla$-differentiable). By $\Delta$ we denote the delta-differentiation operator which assigns to each $\Delta$-differentiable function $f: T \rightarrow \mathbb{R}$ its delta derivative $\Delta(f)$ defined by

$$
[\Delta(f)](x)=f^{\Delta}(x) \quad \text { for } x \in \mathbb{T}^{\kappa} .
$$

The shift operator $E$ is defined by the formula

$$
(E f)(x)=f(\sigma(x))
$$

for $x \in \mathbb{T}$, where $\sigma: T \rightarrow T$ is the forward jump operator. It is convenient, in the operator relations to denote the delta-differentiation operator by $\delta$ rather than by $\Delta$. For example, $\delta f$ will denote the composition (product) of the delta-differentiation operator $\delta$ and the operator of multiplication by the function $f$. According to formula (16) we have

$$
\delta f=f^{\Delta}+E(f) \delta .
$$

Consider the $N$ th order $\delta$-differential operator given by

$$
L=a_{N} \delta^{N}+a_{N-1} \delta^{N-1}+\cdots+a_{1} \delta+a_{0},
$$

where the coefficients $a_{i}(i=0,1, \ldots, N)$ are some $\Delta$-smooth functions of the variable $x \in T$ T. These functions are assumed to depend also on a continuous variable $t \in \mathbb{R}$, however, we will not (for simplicity) indicate explicitly the dependence on $t$.

Proposition 8: Let $L$ be given as in (39) and $A_{n}=\left(L^{n}\right)_{>0}$ be the operator $L^{n}$ missing the $\delta^{0}$ 
term. Then the Lax equation

$$
\frac{\mathrm{d} L}{\mathrm{~d} t_{n}}=\left[A_{n}, L\right]=A_{n} L-L A_{n}
$$

for $n=1,2, \ldots$ produces a consistent hierarchy of coupled nonlinear evolutionary equations.

Example 4. Burgers equation on time scale: Let $L=v \delta+u$, where $u$ and $v$ are functions of $x$ $\in \mathrm{T}$ and $t \in \mathbb{R}$. Then for an appropriate operator $A$ the Lax equation

$$
\frac{\mathrm{d} L}{\mathrm{~d} t}=[A, L]
$$

defines a system of two differential equations for the functions $u$ and $v$. We find the operator $A$ by using the Gelfand-Dikii formalism. Let us start with the second power of $L$ and assume $A$ $=\left(L^{2}\right)_{>0}$, where

$$
L^{2}=v E(v) \delta^{2}+\left[v v^{\Delta}+v E(u)+u v\right] \delta+v u^{\Delta}+u^{2} .
$$

We can assume $A=-\left(L^{2}\right)_{0}$ (the part of $-L^{2}$ without the $\delta$ terms). With this choice, (41) gives

$$
\begin{gathered}
\frac{\mathrm{d} v}{\mathrm{~d} t}=\mu v\left(v u^{\Delta}+u^{2}\right)^{\Delta} \\
\frac{\mathrm{d} u}{\mathrm{~d} t}=v\left(v u^{\Delta}+u^{2}\right)^{\Delta}
\end{gathered}
$$

where $\mu(x)=\sigma(x)-x$ for $x \in \mathbb{T}$.

Equations (43) and (44) given above are not independent of each other. It is easy to see that $v=\mu u+\lambda$, where $\lambda$ is an arbitrary real function depending only on $x \in \mathbb{T}$. Then these two equations reduce to a single equation, a Burgers equation on time scales,

$$
\frac{\mathrm{d} u}{\mathrm{~d} t}=(\mu u+\lambda)\left[u^{2}+(\mu u+\lambda) u^{\Delta}\right]^{\Delta} .
$$

Let us present some special cases: (i) When $\mathbb{T}=\mathbb{R}$ then $\mu=0$ and $\delta=D$, the usual differentiation. Hence we can let $\lambda=1$ and (45) reduces to the standard Burgers equation on R. (ii) When $\mathbb{T}$ $=h Z$ then $\mu(m)=h$ and $f^{\Delta}(m)=(1 / h)[f(m+h)-f(m)]$ for any $f$. Then taking $\lambda=0$ in (45) we find

$$
\frac{\mathrm{d} u(m)}{\mathrm{d} t}=u(m) u(m+h)[u(m+2 h)-u(m)]
$$

where $m \in h Z$. The evolution equation given above in (46) represents a difference version of the Burgers equation. (iii) Let $\mathbb{T}=q^{Z}$, where $q \neq 1$ and $q>0$. Then we have $\mu(x)=(q-1) x$ and $f^{\Delta}(x)$ $=[f(q x)-f(x)] /(q-1) x$ and taking $\lambda=0$ we get from (45)

$$
\frac{\mathrm{d} u(x)}{\mathrm{d} t}=u(x) u(q x)\left[u\left(q^{2} x\right)-u(x)\right] .
$$

Taking $A_{n}=-\left(L^{n}\right)_{0}$ with $L$ given as in Example 4 we get a hierarchy of evolution equations (Burgers hierarchy on time scales) from

$$
\frac{\mathrm{d} L}{\mathrm{~d} t_{n}}=-\left[\left(L^{n}\right)_{0}, v \delta+u\right]
$$

for all $n=1,2,3, \ldots$. Since $\left(L^{n}\right)_{0}$ is a scalar function, letting $\left(L^{n}\right)_{0}=\rho_{n}$ we obtain 


$$
\begin{gathered}
\frac{\mathrm{d} v}{\mathrm{~d} t_{n}}=\mu v\left(\rho_{n}\right)^{\Delta}, \\
\frac{\mathrm{d} u}{\mathrm{~d} t_{n}}=v\left(\rho_{n}\right)^{\Delta},
\end{gathered}
$$

where the first three $\rho_{n}$ are given by

$$
\begin{gathered}
\rho_{1}=u, \\
\rho_{2}=v u^{\Delta}+u^{2}, \\
\rho_{3}=v E(v) u^{\Delta \Delta}+\left[v v^{\Delta}+v E(u)+u v\right] u^{\Delta}+\left(v u^{\Delta}+u^{2}\right) u .
\end{gathered}
$$

The above hierarchy reduces to a single evolution equation with $v=\mu u+\lambda$,

$$
\frac{\mathrm{d} u}{\mathrm{~d} t_{n}}=(\mu u+\lambda)\left(\widetilde{\rho}_{n}\right)^{\Delta}, \quad n=1,2, \ldots,
$$

where $\widetilde{\rho}_{n}$ is equal to $\rho_{n}$ with $v=\mu u+\lambda$. When T is a regular-discrete time scale, the first three $\widetilde{\rho}_{n}$ are given for $\lambda=0$ by

$$
\begin{gathered}
\tilde{\rho}_{1}=u, \\
\widetilde{\rho}_{2}=u E(u), \\
\widetilde{\rho}_{3}=u E(u) E^{2}(u) .
\end{gathered}
$$

It is possible to construct the recursion operator $\mathcal{R}$ by using the Lax representation. ${ }^{14-16}$ The hierarchy satisfies a recursion relation like

$$
\frac{\mathrm{d} L}{\mathrm{~d} t_{n+1}}=L \frac{\mathrm{d} L}{\mathrm{~d} t_{n}}+\left[R_{n}, L\right], \quad n=1,2, \ldots,
$$

where $R_{n}$ is the remainder operator which has the same degree as the Lax operator $L$. We shall construct this operator for the Burgers equation with $\lambda=0$ on regular-discrete time scales. Choosing $R_{n}=\alpha_{n} \delta$ we get [by choosing $v(x)=\mu(x) u(x)$ ]

$$
\mathcal{R}=u E+[u(E(u)-u)](E-1)^{-1} \frac{E}{E(u)} .
$$

One can generate the hierarchy (54) by application of the recursion operator $\mathcal{R}$ to the lowest order symmetry $u_{1}=u(E(u)-u)$,

$$
\frac{\mathrm{d} u}{\mathrm{~d} t_{n}}=\mathcal{R}^{n-1} u_{1}, \quad n=1,2, \ldots
$$

\section{ALGEBRA OF PSEUDO-DELTA-DIFFERENTIAL OPERATORS ON REGULAR TIME SCALES}

Let us define the notion of regular time scales.

Definition 9: We say that a time scale $\mathbb{T}$ is regular if the following two conditions are satisfied simultaneously: 


$$
\text { (i) } \sigma(\rho(x))=x \quad \text { for all } x \in \mathbb{T}
$$

and

$$
\text { (ii) } \rho(\sigma(x))=x \quad \text { for all } x \in \mathbb{T} \text {, }
$$

where $\sigma$ and $\rho$ denote the forward and backward jump operators, respectively.

From (61) it follows that the operator $\sigma: \mathbb{T} \rightarrow \mathbb{T}$ is "onto" while (62) implies that $\sigma$ is "oneto-one." Therefore $\sigma$ is invertible and $\sigma^{-1}=\rho$. Similarly, the operator $\rho: \mathbb{T} \rightarrow \mathbb{T}$ is invertible and $\rho^{-1}=\sigma$ if $\mathrm{T}$ is regular.

Let us set $x_{*}=\min \mathrm{T}$ if there exists a finite $\min \mathrm{T}$, and set $x_{*}=-\infty$ otherwise. Also set $x^{*}$ $=\max \mathrm{T}$ if there exists a finite $\max \mathrm{T}$, and $x^{*}=\infty$ otherwise. It is not difficult to see that the following statement holds.

Proposition 10: A time scale $\mathrm{T}$ is regular if and only if the following two conditions hold:

(i) The point $x_{*}=\mathbb{T}$ is right-dense and the point $x^{*}=\max \mathbb{T}$ is left-dense.

(ii) Each point of $\mathbb{T} \backslash\left\{x_{*}, x^{*}\right\}$ is either two-sided dense or two-sided scattered.

In particular, $\mathbb{R}, h \mathbb{Z}$, and $\mathbb{K}_{q}$ are regular time scales, as are $[0,1],[-1,0] \cup\{1 / k: k$ $\in \mathbb{N}\} \cup\{k /(k+1): k \in \mathbb{N}\} \cup[1,2]$, and $(-\infty, 0] \cup\{1 / k: k \in \mathbb{N}\} \cup\{2 k: k \in \mathbb{N}\}$, where [-1,0], [0,1], $[1,2],(-\infty, 0]$ are real line intervals.

If $f: \mathbb{T} \rightarrow \mathbb{R}$ is a function we define the functions $f^{\sigma}: \mathbb{T} \rightarrow \mathbb{R}$ and $f^{\rho}: \mathbb{T} \rightarrow \mathbb{R}$ by

$$
f^{\sigma}(x)=f(\sigma(x)) \quad \text { and } f^{\rho}(x)=f(\rho(x)) \text { for all } x \in \mathbb{T} \text {. }
$$

Defining the shift operator $E$ by the formula $E f=f^{\sigma}$ we have

$$
(E f)(x)=f^{\sigma}(x)=f(\sigma(x)) \text { for all } x \in \mathbb{T} .
$$

The inverse $E^{-1}$ exists only in case of regular time scales and is defined by

$$
\left(E^{-1} f\right)(x)=f\left(\sigma^{-1}(x)\right)=f(\rho(x)) \quad \text { for all } x \in \mathbb{T} .
$$

In the operator relations, for convenience, we will denote the shift operator by $\mathcal{E}$ rather than by $E$. For example, $\mathcal{E} f$ will denote the composition (product) of the shift operator $\mathcal{E}$ and the operator of multiplication by the function $f$. Obviously, for any integer $m \in \mathbb{Z}$, we have

$$
\mathcal{E}^{m} f=\left(E^{m} f\right) \mathcal{E}^{m}
$$

Remember that $\delta$ denotes the delta-differentiation operator acting in the operator relations by $\delta f=f^{\Delta}+E(f) \delta$. The following proposition is an immediate consequence of the formulas (8) and (16).

Proposition 11: The operator formulas

$$
\mathcal{E}=I+\mu \delta
$$

and

$$
\delta f=f^{\Delta}+E(f) \delta
$$

hold, where the function $\mu: \mathbb{T} \rightarrow \mathbb{R}$ is defined by $\mu(x)=\sigma(x)-x$ for all $x \in \mathbb{T}$, and I denotes the identity operator.

In this section we will assume that all our considered functions from $\mathrm{T}$ to $\mathrm{R}$ are $\Delta$-smooth and tend to zero sufficiently rapidly together with their $\Delta$-derivatives as $x$ goes to $x_{*}$ or $x^{*}$, where $x_{*}$ $=\min \mathbb{T}$ if there exists a finite $\min \mathbb{T}$ and $x_{*}=-\infty$ otherwise, $x^{*}=\max \mathbb{T}$ if there exists a finite $\max \mathbb{T}$ and $x^{*}=\infty$ otherwise. The inverse operator $\delta^{-1}$ exists on such functions. If $g: T \rightarrow T$ is such a function, then 


$$
\left[\Delta^{-1}(g)\right](x)=\int_{x_{*}}^{x} g(y) \Delta y .
$$

Proposition 12: Let $f: \mathbb{T} \rightarrow \mathbb{R}$ be a $\Delta$-smooth function such that $f$ and all its $\Delta$-derivatives vanish rapidly at $x_{*}$ and $x^{*}$. Then the operator $\delta^{-1} f$ being the composition (product) of $\delta^{-1}$ and $f$ has the form of the formal series in powers of $\delta^{-1}$,

$$
\delta^{-1} f=\alpha_{0} \delta^{-1}+\alpha_{1} \delta^{-2}+\cdots,
$$

where $\alpha_{0}=E^{-1} f$, and $\alpha_{k}=(-1)^{k}\left(E^{-1} f\right)^{\nabla^{k}}$ for $k=1,2, \ldots$.

Proof: Multiplying (68) on the left and right by $\delta^{-1}$ we obtain

$$
\delta^{-1} E(f)=f \delta^{-1}-\delta^{-1} f^{\Delta} \delta^{-1} .
$$

Replacing here $f$ by $E^{-1} f$ we get

$$
\delta^{-1} f=\left(E^{-1} f\right) \delta^{-1}-\delta^{-1}\left(E^{-1} f\right)^{\Delta} \delta^{-1} .
$$

Further, applying this rule to the function $\left(E^{-1} f\right)^{\Delta}$ and taking into account that by Proposition 4(i)

$$
E^{-1}\left(E^{-1} f\right)^{\Delta}=\left(E^{-1} f\right)^{\nabla},
$$

we find

$$
\delta^{-1}\left(E^{-1} f\right)^{\Delta}=\left(E^{-1} f\right)^{\nabla} \delta^{-1}-\delta^{-1}\left(\left(E^{-1} f\right)^{\nabla}\right)^{\Delta} \delta^{-2} .
$$

Substituting this into the second term on the right-hand side of (72) we obtain

$$
\delta^{-1} f=\left(E^{-1} f\right) \delta^{-1}-\left(E^{-1} f\right)^{\nabla} \delta^{-2}+\delta^{-1}\left(\left(E^{-1} f\right)^{\Delta}\right)^{\nabla} \delta^{-2} .
$$

Continuing this procedure repeatedly we arrive at the statement of the proposition.

Definition 13: By $\Lambda$ we denote the algebra of pseudo-delta-differential operators. Any operator $K \in \Lambda$ of order $k$ has the form

$$
K=\sum_{\ell=-\infty}^{k} a_{\ell} \delta^{\ell}
$$

where $a_{\ell}$ 's are $\Delta$-smooth functions of $x \in \mathbb{T}$. For $K$ given by (76) we will use the following notations:

$$
K_{\geqslant 0}=\sum_{\ell=0}^{k} a_{\ell} \delta^{\ell} \quad \text { and } K_{<0}=\sum_{-\infty}^{-1} a_{\ell} \delta^{\ell}
$$

As an example we let

$$
L=a_{N} \delta^{N}+a_{N-1} \delta^{N-1}+\cdots+a_{1} \delta+a_{0},
$$

where $a_{i}(i=0,1, \ldots, N)$ are some $\Delta$-smooth functions on T. Then we have the following.

Proposition 16: Let $L$ be given in (78). For each fixed $N$ the Lax equation

$$
\frac{\mathrm{d} L}{\mathrm{~d} t_{n}}=\left[A_{n}, L\right], \quad A_{n}=\left(L^{n / N}\right)_{\geqslant 0},
$$

for $n=1,2, \ldots$ not divisible by $N$, produces a (consistent) hierarchy of evolution equations ( $a \mathrm{KdV}$ hierarchy on time scales).

Proof: Since $\left(L^{n / N}\right)_{\geqslant 0}=L^{n / N}-\left(L^{n / N}\right)_{<0}$, we get 


$$
\left.\frac{\mathrm{d} L}{\mathrm{~d} t_{n}}=\left[\left(L^{n / N}\right)_{\geqslant 0}, L\right]=-\left[L^{n / N}\right)_{<0}, L\right] .
$$

Evidently the commutator $\left[\left(L^{n / N}\right)_{\geqslant 0}, L\right]$ involves only non-negative powers of $\delta$, while the commutator $\left[\left(L^{n / N}\right)_{<0}, L\right]$ has the form $\Sigma_{j=-\infty}^{N-1} b_{j} \delta^{j}$. Therefore, we get by (80) that, for all $n$ not divisible by $N$, (79) produces nontrivial consistent $N+1$-number of evolutionary coupled $\Delta$-differential equations for $a_{i}, i=0,1, \ldots, N$. Note that $a_{N}$ turns out to be a fixed (i.e., time independent) function of $x$.

Example 5: A KdV hierarchy on time scales. Let

$$
L=\delta^{2}+v \delta+u,
$$

where $u$ and $v$ are $\Delta$-smooth functions. It is straightforward to find that

$$
L^{1 / 2}=\delta+\alpha_{0}+\alpha_{1} \delta^{-1}+\alpha_{2} \delta^{-2}+\cdots
$$

where

$$
\begin{gathered}
E\left(\alpha_{0}\right)+\alpha_{0}=v, \\
E\left(\alpha_{1}\right)+\alpha_{1}+\left(\alpha_{0}\right)^{\Delta}+\left(\alpha_{0}\right)^{2}=u, \\
E\left(\alpha_{2}\right)+\alpha_{2}+\alpha_{1} E^{-1}\left(\alpha_{0}\right)+\left(\alpha_{1}\right)^{\Delta}=0 .
\end{gathered}
$$

Choosing $n=1,3, \ldots$ we get the members of the KdV hierarchy.

(1) Let $n=1$. Then Lax equation (79) becomes

$$
\frac{\mathrm{d} v}{\mathrm{~d} t} \delta+\frac{\mathrm{d} u}{\mathrm{~d} t}=\left[\left(L^{1 / 2}\right)_{\geqslant 0}, L\right]
$$

and gives coupled equations for $u$ and $v$,

$$
\begin{gathered}
\frac{\mathrm{d} u}{\mathrm{~d} t}=u^{\Delta}-v\left(\alpha_{0}\right)^{\Delta}-\left(\alpha_{0}\right)^{\Delta \Delta}, \\
\frac{\mathrm{d} v}{\mathrm{~d} t}=v^{\Delta}+E(u)-u-v\left[E\left(\alpha_{0}\right)-\alpha_{0}\right]-E\left(\alpha_{0}^{\Delta}\right)-E\left(\alpha_{0}\right)^{\Delta}=\mu\left(u^{\Delta}-v\left(\alpha_{0}\right)^{\Delta}-\left(\alpha_{0}\right)^{\Delta \Delta}\right) .
\end{gathered}
$$

Comparing the above equations we get

$$
\frac{\mathrm{d} v}{\mathrm{~d} t}-\mu \frac{\mathrm{d} u}{\mathrm{~d} t}=0
$$

and therefore

$$
v=\mu u+\lambda,
$$

where $\lambda$ is an arbitrary real function depending only on $x \in \mathbb{T}$. Thus, two equations (87) and (88) reduce to the following single equation:

$$
\frac{\mathrm{d} u}{\mathrm{~d} t}=u^{\Delta}-(\mu u+\lambda)\left(\alpha_{0}\right)^{\Delta}-\left(\alpha_{0}\right)^{\Delta \Delta},
$$

where $\alpha_{0}$ is expressed, according to (83), from 


$$
E\left(\alpha_{0}\right)+\alpha_{0}=\mu u+\lambda .
$$

If we take $\lambda=0$, then (91) and (92) become

$$
\begin{gathered}
\frac{\mathrm{d} u}{\mathrm{~d} t}=u^{\Delta}-\mu u\left(\alpha_{0}\right)^{\Delta}-\left(\alpha_{0}\right)^{\Delta \Delta}, \\
E\left(\alpha_{0}\right)+\alpha_{0}=\mu u .
\end{gathered}
$$

We shall now give $\alpha_{0}$, for illustration, for particular cases of $T$.

(i) In the case $\mathbb{T}=\mathbb{R}$ we have $\mu=0$ and (94) gives $\alpha_{0}=0$ and (93) becomes

$$
\frac{\mathrm{d} u}{\mathrm{~d} t}=\frac{\mathrm{d} u}{\mathrm{~d} x},
$$

which is a linear equation explicitly solvable,

$$
u(x, t)=\varphi(x+t),
$$

where $\varphi$ is an arbitrary differentiable function.

(ii) In the case $\mathbb{T}=\mathbb{Z}$ we have $\mu=1$ and (94) is satisfied by

$$
\alpha_{0}(n)=-\sum_{k=-\infty}^{n-1}(-1)^{n+k} u(k), \quad n \in \mathbb{Z}
$$

and therefore the Eq. (93) becomes

$$
\frac{\mathrm{d} u(n)}{\mathrm{d} t}=-u^{2}(n)+2 u(n)+2(-1)^{n}[2+u(n)] \sum_{k=-\infty}^{n-1}(-1)^{k} u(k),
$$

for $n \in \mathbb{Z}$.

(iii) In the case $\mathbb{T}=\mathbb{K}_{q}$ we have $\mu(x)=(q-1) x$ and (94) is satisfied by $\alpha_{0}(0)=0$ and

$$
\alpha_{0}(x)=-(q-1) \sum_{y \in\left(0, q^{-1} x\right]}(-1)^{\log _{q}(x y)} y u(y)
$$

for $x \in \mathbb{K}_{q}$ and $x \neq 0$. Substituting (99) into (93) we can get an evolution equation for $u$.

(iv) Let $\mathbb{T}=(-\infty, 0) \cup \mathbb{K}_{q}=(-\infty, 0] \cup q^{Z}$. In this case $\mu(x)=0$ if $x \in(-\infty, 0]$ and $\mu(x)=(q$ $-1) x$ if $x \in q^{Z}$. The equation (94) is satisfied by the function $\alpha_{0}$ given by

$$
\alpha_{0}(x)=\left\{\begin{array}{l}
0 \quad x \in(-\infty, 0], \\
-(q-1) \Sigma_{y \in\left(0, q^{-1} x\right]}(-1)^{\log _{q}(x y)} y u(y) \quad x \in q^{Z} .
\end{array}\right.
$$

Therefore (93) will yield an evolution equation coinciding on $(-\infty, 0]$ and $q^{Z}$ with the evolution equations described in the examples (i) and (iii), respectively. Now an essential complementary point is that the solution $u$ must satisfy at $x=0$ the smoothness conditions

$$
u\left(0^{-}\right)=u\left(0^{+}\right), \quad u^{\prime}\left(0^{-}\right)=u^{\Delta}\left(0^{+}\right) .
$$

(2) Letting $n=3$, first we get

$$
L^{3 / 2}=\delta^{3}+p \delta^{2}+q \delta+r+(\text { terms with negative powers of } \delta),
$$

where

$$
p=\alpha_{0}+E(v)
$$




$$
\begin{gathered}
q=v^{\Delta}+E(u)+\alpha_{0} v+\alpha_{1}, \\
r=u^{\Delta}+\alpha_{0} u+\alpha_{1} E^{-1}(v)+\alpha_{2},
\end{gathered}
$$

and the Lax equation

$$
\frac{\mathrm{d} v}{\mathrm{~d} t} \delta+\frac{\mathrm{d} u}{\mathrm{~d} t}=\left[\left(L^{3 / 2}\right)_{\geqslant 0}, L\right],
$$

gives the coupled equations for $u$ and $v$,

$$
\begin{gathered}
\frac{\mathrm{d} u}{\mathrm{~d} t}=u^{\Delta \Delta \Delta}+p u^{\Delta \Delta}+q u^{\Delta}-r^{\Delta \Delta}-v r^{\Delta} \\
\frac{\mathrm{d} v}{\mathrm{~d} t}=v^{\Delta \Delta \Delta}+E\left(u^{\Delta \Delta}\right)+\left(E\left(u^{\Delta}\right)\right)^{\Delta}+E(u)^{\Delta \Delta}+p\left[v^{\Delta \Delta}+E\left(u^{\Delta}\right)+E(u)^{\Delta}\right]+q\left(v^{\Delta}+E(u)-u\right)+r v-q^{\Delta \Delta} \\
-E\left(r^{\Delta}\right)-E(r)^{\Delta}-v q^{\Delta}-v E(r) .
\end{gathered}
$$

As in the first member of the hierarchy ( $n=1$ case), the above $\Delta-\mathrm{KdV}$ equations reduce to a single equation for the function $u$. Below in Corollary 23 we found that $v=\mu(x) u+\lambda(x)$. Letting $\lambda=$ constant we get

$$
\frac{\mathrm{d} u}{\mathrm{~d} t}=u^{\Delta \Delta \Delta}+p u^{\Delta \Delta}+q u^{\Delta}-r^{\Delta \Delta}-v r^{\Delta} .
$$

It is possible to write the above equation more explicitly in terms of $u$ for $\mathbb{T}=\mathbb{R}, \mathbb{T}=\mathbb{Z}$, and for $\mathbb{T}=\mathbb{K}_{q}$ but they are quite lengthy. For the discrete case we give a $\mathrm{KdV}$ hierarchy in Example 8, next section.

\section{SHIFT LAX OPERATORS ON REGULAR-DISCRETE TIME SCALES}

Let $\mathrm{T}$ be a time scale. Let us set $x_{*}=\min \mathrm{T}$ if there exists a finite min $\mathrm{T}$ and $x_{*}=-\infty$ otherwise. Also set $x^{*}=\max \mathbb{T}$ if there exists a finite $\max \mathbb{T}$ and $x^{*}=\infty$ otherwise. We will briefly write $x_{*}$ $=\min \mathbb{T}$ and $x^{*}=\max \mathbb{T}$.

Definition 17: We say that a time scale $\mathbb{T}$ is regular-discrete if the following two conditions are satisfied:

(i) The point $x_{*}$ is right-dense and the point $x^{*}$ is left-dense.

(ii) Each point of $\mathbb{T} \backslash\left\{x_{*}, x^{*}\right\}$ is two-sided scattered (isolated).

The shift operator $E$ is defined on functions $f: \mathbb{T} \rightarrow \mathbb{R}$ by the formula

$$
(E f)(x)=f(\sigma(x)) \quad \text { for } x \in \mathbb{T},
$$

where $\sigma: \mathrm{T} \rightarrow \mathrm{T}$ is the forward jump operator.

In this section we deal only with regular-discrete time scales $\mathbb{T}$. For such time scales $T$ we have

$$
\mu(x)=\sigma(x)-x \neq 0 \quad \text { for all } x \in \mathbb{T} \backslash\left\{x_{*}, x^{*}\right\}
$$

and, therefore, on functions given on $\mathbb{T} \backslash\left\{x_{*}, x^{*}\right\}$ we have the operator relationship

$$
\delta=\frac{1}{\mu}(\mathcal{E}-1) .
$$

All our functions will be assumed to be defined on $T \backslash\left\{x_{*}, x^{*}\right\}$ and tends to zero sufficiently rapidly as $x$ goes to $x_{*}$ or $x^{*}$. 
This shift operator $\mathcal{E}$, should be quite useful in the application of the Gel'fand-Dikii formalism. The reason is that for any integer $m$ we have the simple product rule

$$
\mathcal{E}^{m} u=\left(E^{m} u\right) \mathcal{E}^{m} .
$$

Hence, for regular-discrete time scales, we can define an algebra of $\mathcal{E}$ operators.

Definition 18: An algebra, $\Lambda_{\epsilon}$ of $\mathcal{E}$ operators satisfying the operator equation (113) is defined as follows: Any operator $K$ in $\Lambda_{\epsilon}$ with degree $k$ is of the form

$$
K=\sum_{-\infty}^{k} a_{\ell} \mathcal{E}^{\ell}
$$

where $a_{\ell}$ are functions of $x \in \mathbb{T}$ that depend also on $t \in \mathbb{R}$.

Hence we can form Lax operators in $\Lambda_{\epsilon}$, and produce integrable equations on regular-discrete time scales. Following ${ }^{3}$ we obtain two classes of Lax representations.

Proposition 19: The Lax equation

$$
\frac{\mathrm{d} L}{\mathrm{~d} t_{\ell}}=\left[\left(L^{\ell}\right)_{\geqslant k}, L\right], \quad k=0,1
$$

produces consistent hierarchy of equations for $\ell=1,2, \ldots$ with the following suitable Lax operators:

$$
\begin{gathered}
L=\mathcal{E}^{\alpha+n}+u_{\alpha+n-1} \mathcal{E}^{\alpha+n-1}+\cdots+u_{\alpha} \mathcal{E}^{\alpha}, \\
L=v_{\alpha+n} \mathcal{E}^{\alpha+n}+v_{\alpha+n-1} \mathcal{E}^{\alpha+n-1}+\cdots+v_{\alpha+1} \mathcal{E}^{\alpha+1}+E^{\alpha},
\end{gathered}
$$

for $k=0$ and $k=1$, respectively. Here $u_{i}$ and $v_{i}$ are functions defined on $\mathrm{T}$ and the integer $\alpha$ is restricted to satisfy the inequality $-n<\alpha \leqslant-1$.

Remark: Lax operators above and the following examples are given on any regular-discrete time scale $\mathbb{T}$ (we can take in particular $\mathbb{T}=\mathbb{Z}$ or $\mathbb{K}_{q}$ ). This means that for any function $u$ on such a time scale $E(u)=u(\sigma(x))$ where $\sigma$ is the jump operator defined in the second section. Hence our examples and results should be considered as more general than those considered in Ref. 3. In the case of Ref. 3 time scale is just the integers $(\mathbb{T}=\mathbb{Z})$ where $E(u(n))=u(n+1)$.

Example 6: Two field equations. Let $k=0, \alpha=-1$ and

$$
L=u_{-1} \mathcal{E}^{-1}+u_{0}+\mathcal{E} \equiv v \mathcal{E}^{-1}+u+\mathcal{E} .
$$

Then we find

$$
\begin{gathered}
\ell=1 \quad \frac{\mathrm{d} v}{\mathrm{~d} t_{1}}=v\left(u-E^{-1}(u)\right), \\
\frac{\mathrm{d} u}{\mathrm{~d} t_{1}}=E(v)-v, \\
\ell=2 \quad \frac{\mathrm{d} v}{\mathrm{~d} t_{2}}=u^{2} v+E(v) v-v E^{-1}(v)-v E^{-1}\left(u^{2}\right), \\
\frac{\mathrm{d} u}{\mathrm{~d} t_{2}}=u E(v)+E(u) E(v)-v E^{-1}(u)-u v,
\end{gathered}
$$




$$
\begin{aligned}
\ell= & 3, \quad \frac{\mathrm{d} v}{\mathrm{~d} t_{3}}=u v^{2}+u^{3} v-v E^{-1}(v) E^{-2}(u)-2 v E^{-1}(u) E^{-1}(v)-v E^{-1}\left(u^{3}\right)+2 u v E(v)-v^{2} E^{-1}(u) \\
& +v E(u) E(v) \\
\frac{\mathrm{d} u}{\mathrm{~d} t_{3}}= & E(v)\left[u^{2}+u E(u)+E\left(u^{2}\right)+E(v)+\left(E^{2}(v)\right)\right]-v\left[E^{-1}(v)+E^{-1}\left(u^{2}\right)+u E^{-1}(u)+u^{2}+v\right]
\end{aligned}
$$

This is a Toda hierarchy on discrete time scales. The recursion relation between the $n+1$ th and $n$th elements of the hierarchy is given by

$$
\begin{aligned}
& v_{n+1}=u v_{n}+v u_{n}+v E^{-1}\left(u_{n}\right)+v\left(E^{-1}(u)-u\right)(1-E)^{-1} \frac{v_{n}}{v}, \\
& u_{n+1}=E\left(v_{n}\right)+u u_{n}+v(1-E)^{-1} \frac{v_{n}}{v}-E(v)(1-E)^{-1} E \frac{v_{n}}{v} .
\end{aligned}
$$

From this recursion relation the recursion operator of the hierarchy follows.

Example 7: Four-field system on time scale. We give two examples which are studied in Ref. 3 for the case $\mathbb{T}=\mathbb{Z}$.

(1) Let $k=0$ and $\alpha=-2$ and

$$
L=\mathcal{E}^{2}+w \mathcal{E}+v+u \mathcal{E}^{-1}+p \mathcal{E}^{-2} .
$$

Then we get the four-field equations

$$
\begin{gathered}
\ell=1 \quad \frac{\mathrm{d} p}{\mathrm{~d} t_{1}}=v p-p E^{-2}(v), \\
\frac{\mathrm{d} u}{\mathrm{~d} t_{1}}=v u+w E(p)-p E^{-2}(w)-u E^{-1}(v), \\
\frac{\mathrm{d} v}{\mathrm{~d} t_{1}}=w E(u)+E^{2}(p)-u E^{-1}(w)-p \\
\frac{\mathrm{d} w}{\mathrm{~d} t_{1}}=E^{2}(u)-u
\end{gathered}
$$

(2) Let $k=1$ and $\alpha=-2$ and

$$
L=\bar{q} \mathcal{E}^{2}+\bar{w} \mathcal{E}+\bar{v}+\bar{u} \mathcal{E}^{-1}+\mathcal{E}^{-2} .
$$

Then we get another four-field equations,

$$
\begin{gathered}
\ell=1 \quad \frac{\mathrm{d} \bar{u}}{\mathrm{~d} t_{1}}=\bar{w}-E^{-2}(\bar{w}), \\
\frac{\mathrm{d} \bar{v}}{\mathrm{~d} t_{1}}=\bar{w} E(\bar{u})+\bar{q}-E^{-2}(\bar{q})-\bar{u} E^{-1}(\bar{w}),
\end{gathered}
$$




$$
\begin{gathered}
\frac{\mathrm{d} \bar{w}}{\mathrm{~d} t_{1}}=\bar{w} E(\bar{v})+\bar{q} E^{2}(\bar{u})-\bar{u} E^{-1}(\bar{q})-\bar{v} \bar{w}, \\
\frac{\mathrm{d} \bar{q}}{\mathrm{~d} t_{1}}=\bar{q} E^{2}(\bar{v})-\bar{v} \bar{q} .
\end{gathered}
$$

So far we considered the hierarchies coming from Proposition 19 with integer powers of the Lax operators. Now we consider the rational powers of the Lax operator.

Proposition 22: Let

$$
L=w \mathcal{E}^{N}+u_{N-1} \mathcal{E}^{N-1}+\cdots+u_{0},
$$

where $w(x)$ is a function of $x$ which is not a dynamical variable $\mathrm{d} w / \mathrm{d} t=0, u_{i}, i=0,1, \ldots, N-1$ are functions of $t$ and $x \in \mathbb{T}$. Then

$$
\frac{\mathrm{d} L}{\mathrm{~d} t_{n}}=\left[\left(L^{n / N}\right)_{\geqslant 0}, L\right], \quad n=1,2, \ldots
$$

produces hierarchies of integrable systems. Here $n$ is a positive integer not divisible by N. Furthermore the function $u_{0}$ is also not dynamical, i.e., $u_{0}=u_{0}(x)$, not depending on $t$.

Corollary 23: When $N=2$ and $w=(1 / \mu) E(1 / \mu)$ then the $\Delta-K d V$ Lax operator (81) reduces to the above form with

$$
\begin{gathered}
u_{0}=-\frac{v}{\mu}+\frac{1}{\mu^{2}}+u, \\
u_{1}=-\frac{1}{\mu}\left[E\left(\frac{1}{\mu}\right)+\frac{1}{\mu}\right]+\frac{v}{\mu} .
\end{gathered}
$$

Hence in part (2) of Example 5 we have a single equation with $v=-\mu u_{0}+(1 / \mu)+\mu u$.

In the following example we study the $N=2$ case in more detail.

Example 8: $\mathrm{KdV}$ on discrete time scales. Let

$$
L=w E(w) \mathcal{E}^{2}+u \mathcal{E}+v .
$$

Then

$$
L^{1 / 2}=w \mathcal{E}+\alpha_{0}+\alpha_{1} \mathcal{E}^{-1}+\alpha_{2} \mathcal{E}^{-2}+\cdots,
$$

where first three $\alpha_{i}$ are given as

$$
\begin{gathered}
w\left(E\left(\alpha_{0}\right)+\alpha_{0}\right)=u, \\
w E\left(\alpha_{1}\right)+E^{-1}(w) \alpha_{1}=v-\left(\alpha_{0}\right)^{2}, \\
w E\left(\alpha_{2}\right)+E^{-2}(w) \alpha_{2}=-\frac{\alpha_{1} E^{-1}(u)}{E^{-1}(w)} .
\end{gathered}
$$

Then we calculate $L^{3 / 2}$ by

$$
L^{3 / 2}=w E(w) E^{2}(w) \mathcal{E}^{3}+p_{2} \mathcal{E}^{2}+p_{1} \mathcal{E}+p_{0}+\text { negative powers of } \mathcal{E},
$$

where

$$
p_{2}=E(w)\left[w E^{2}\left(\alpha_{0}\right)+u\right],
$$




$$
\begin{gathered}
p_{1}=w E(w) E^{2}\left(\alpha_{1}\right)+u E\left(\alpha_{0}\right)+w v, \\
p_{0}=w E(w) E^{2}\left(\alpha_{2}\right)+u E\left(\alpha_{1}\right)+v \alpha_{0}, \\
=w E^{-1}(w)\left[E^{-1}(w)+E(w) E\right]^{-1}\left(E\left(\alpha_{1}\right) \frac{u}{w}\right)+v(1+E)^{-1} \frac{u}{w} .
\end{gathered}
$$

Then (138) with $N=2$ produces a hierarchy of evolution equations. It turns out that $v$ becomes a constant in the whole hierarchy. We give the first two members of the hierarchy (for $n=1$ and $n$ $=3$ ),

$$
\begin{gathered}
u_{t_{1}}=u(1-E)(1+E)^{-1} \frac{u}{w}, \\
u_{t_{3}}=u(1-E) p_{0},
\end{gathered}
$$

where $p_{0}$ is given above. The next members of the hierarchy can be found by taking $n=5$ in (138) or by applying the recursion operator $\mathcal{R}$ to $u_{t_{3}}$. For $\mathbb{T}=\mathbb{K}_{q}$ and $w=1$ the above hierarchy and its Hamilton formulation were given by Frenkel. ${ }^{4}$ The recursion operator of this hierarchy with $w$ $=1$ can be found by using (58) with $R_{n}=\alpha_{n} \mathcal{E}+\beta_{n}$. We find that

$$
\begin{gathered}
\left(E^{2}-1\right) \alpha_{n}=E^{2}\left(u_{n}\right), \\
\left(E^{2}-1\right) \beta_{n}=u E\left(u_{n}\right)+E(u) \alpha_{n}-u E\left(\alpha_{n}\right)
\end{gathered}
$$

and the equation which determines the recursion operator is

$$
u_{n+1}=v u_{n}-u(E-1) \beta_{n}, \quad n=0,1,2, \ldots .
$$

We find that

$$
\mathcal{R}=v-u(E+1)^{-1}[-u+E(u) E]\left(E^{2}-1\right)^{-1} E .
$$

When the Lax operator is of degree one and has an infinite power series in operator $\mathcal{E}^{-1}$ the corresponding system is called the KP hierarchy.

Proposition 24: Let

$$
L=\mathcal{E}+u_{0}+u_{1} \mathcal{E}^{-1}+u_{2} \mathcal{E}^{-2}+\cdots
$$

Then

$$
\frac{\mathrm{d} L}{\mathrm{~d} t_{n}}=\left[\left(L^{n}\right)_{\geqslant 0}, L\right], \quad n=1,2, \ldots,
$$

produces the following hierarchy:

$$
\begin{gathered}
n=1 \quad \frac{\mathrm{d} u_{0}}{\mathrm{~d} t_{1}}=(E-1) u_{1}, \\
\frac{\mathrm{d} u_{1}}{\mathrm{~d} t_{1}}=(E-1) u_{2}+u_{1}\left[u_{0}-E^{-1}\left(u_{0}\right)\right], \\
\frac{\mathrm{d} u_{k}}{\mathrm{~d} t_{1}}=(E-1) u_{k+1}+u_{k}\left[u_{0}-E^{-k}\left(u_{0}\right)\right], \quad k=0,1, \ldots .
\end{gathered}
$$




$$
\begin{gathered}
n=2 \quad \frac{\mathrm{d} u_{0}}{\mathrm{~d} t_{2}}=\left(E^{2}-1\right) u_{2}-u_{1} E^{-1}(E+1) u_{0}+E\left(u_{1}\right)\left(E\left(u_{0}\right)+u_{0}\right), \\
\frac{\mathrm{d} u_{1}}{\mathrm{~d} t_{2}}=\left(E^{2}-1\right) u_{3}+\alpha_{1} E\left(u_{2}\right)-u_{2} E^{-2}\left(\alpha_{1}\right)+\alpha_{0} u_{1}-u_{1} E^{-1}\left(\alpha_{0}\right), \\
\frac{\mathrm{d} u_{k}}{\mathrm{~d} t_{2}}=\left(E^{2}-1\right) u_{k+2}+\alpha_{1} E\left(u_{k+1}\right)-u_{k+1} E^{-k-1}\left(\alpha_{1}\right)+\alpha_{0} u_{k}-u_{k} E^{-k}\left(\alpha_{0}\right), \quad k=0,1, \ldots,
\end{gathered}
$$

where $\alpha_{0}=(E+1) u_{1}+\left(u_{0}\right)^{2}$ and $\alpha_{1}=(E+1) u_{0}$. The case $\mathbb{T}=\mathbb{Z}$ of this hierarchy is discussed in Ref. 3 (see also the references therein) and the case $\mathbb{T}=\mathbb{K}_{q}$ is discussed in Refs. 4 and 5.

\section{TRACE FUNCTIONAL AND CONSERVATION LAWS}

Let $\mathbb{T}$ be a regular time scale and $\Lambda$ be the algebra of pseudo-delta-differential operators. Any operator $F \in \Lambda$ of order $k$ has the form

$$
F=a_{k} \delta^{k}+a_{k-1} \delta^{k-1}+\cdots+a_{1} \delta+a_{0}+a_{-1} \delta^{-1}+a_{-2} \delta^{-2}+\cdots,
$$

where $a_{\ell}$ 's are $\Delta$-smooth functions of $x \in \mathbb{T}$ (they are also functions of $t \in \mathbb{R}$ ). The coefficients $a_{0}$ and $a_{-1}$ we call, respectively, the free term (zero order term) and the residue of $F$ associated with its " $\delta$-expansion" (165) and write

$$
\text { Free }_{\delta} F=a_{0}(x) \text { and } \operatorname{Res}_{\delta} F=a_{-1}(x) .
$$

In case of regular-discrete time scales $\mathbb{T}$ we have

$$
\delta=\frac{1}{\mu}(\mathcal{E}-I)=\frac{1}{\mu} \mathcal{E}-\frac{1}{\mu}
$$

and therefore the same operator $F$ can be expanded in series with respect to the powers of $\mathcal{E}$ of the form

$$
F=b_{k} \mathcal{E}^{k}+b_{k-1} \mathcal{E}^{k-1}+\cdots+b_{1} \mathcal{E}+b_{0}+b_{-1} \mathcal{E}^{-1}+b_{-2} \mathcal{E}^{-2}+\cdots
$$

We write

$$
\operatorname{Free}_{\mathcal{E}} F=b_{0}(x) \text { and } \operatorname{Res}_{\mathcal{E}} F=b_{-1}(x) .
$$

Substituting (167) and

$$
\delta^{-1}=(\mathcal{E}-I)^{-1} \mu=\left(\mathcal{E}^{-1}+\mathcal{E}^{-2}+\cdots\right) \mu=E^{-1}(\mu) \mathcal{E}^{-1}+E^{-2}(\mu) \mathcal{E}^{-2}+\cdots,
$$

into (165) and taking into account that

$$
E^{-1}(\mu)=\mu(\rho(x))=\sigma(\rho(x))-\rho(x)=x-\rho(x)=\nu(x),
$$

we find that

$$
\operatorname{Res}_{\mathcal{E}} F=\nu \operatorname{Res}_{\delta} F .
$$

Definition 25: The trace of an operator $F \in \Lambda$ is defined by

$$
\operatorname{Tr}(F)=\int_{\mathbb{T}} \operatorname{Res}_{\delta}\left\{F(I+\mu \delta)^{-1}\right\} \nabla x,
$$

where the nabla integral is defined according to Sec. II.

Proposition 26: Let $F$ be given as in (165). In case of regular-discrete time scales we have 


$$
\operatorname{Res}_{\delta}\left\{F(I+\mu \delta)^{-1}\right\}=\frac{1}{\nu(x)} \text { Free }_{\mathcal{E}} F
$$

for $x \in \mathbb{T} \backslash\left\{x_{*}, x^{*}\right\}$, where $x_{*}=\min \mathbb{T}$ and $x^{*}=\max \mathbb{T}$. Therefore in this case

$$
\operatorname{Tr}(F)=\int_{\mathbb{T}}\left(\text { Free }_{\mathcal{E}} F\right) \frac{\nabla x}{\nu(x)}=\sum_{x \in T} b_{0}(x) .
$$

Proof: Since $I+\mu \delta=\mathcal{E}$ we have, by using (172) and (168),

$$
\begin{aligned}
\nu \operatorname{Res}_{\delta}\left\{F(I+\mu \delta)^{-1}\right\} & =\operatorname{Res}_{\mathcal{E}}\left\{F(I+\mu \delta)^{-1}\right\}=\operatorname{Res}_{\mathcal{E}}\left(F \mathcal{E}^{-1}\right)=\operatorname{Res}_{\mathcal{E}}\left(b_{k} \mathcal{E}^{k-1}+\cdots+b_{1}+b_{0} \mathcal{E}^{-1}+\cdots\right) \\
& =b_{0}=\operatorname{Free}_{\mathcal{E}}(F) .
\end{aligned}
$$

Proposition 27: For all $F_{1}, F_{2} \in \Lambda$,

$$
\operatorname{Tr}\left(\left[F_{1}, F_{2}\right]\right)=\operatorname{Tr}\left(F_{1} F_{2}-F_{2} F_{1}\right)=0,
$$

in other words the pairing $\left(F_{1}, F_{2}\right)=\operatorname{Tr}\left(F_{1} F_{2}\right)$ is symmetric.

We prove (177) only for particular cases of time scales $\mathbb{T}$. They indicate a way to the proof in the general case of regular time scales.

(i) If $\mathbb{T}=\mathbb{R}$, then $\delta=\partial=(d / \mathrm{d} x) \cdot$ and $\mu(x)=0$,

$$
F=a_{k} \partial^{k}+\cdots+a_{1} \partial+a_{0}+a_{-1} \partial^{-1}+\cdots
$$

and

$$
\operatorname{Tr}(F)=\int_{\mathbb{R}} \operatorname{Res}(F) \mathrm{d} x=\int_{\mathbb{R}} a_{-1}(x) \mathrm{d} x .
$$

It is well known that (for example, see Ref. 3) for such functional $\operatorname{Tr}(F)$ the statement (177) holds.

(ii) Let $\mathbb{T}$ be a regular-discrete time scale. Then by Proposition 26 we have

$$
\operatorname{Tr}\left(\left[F_{1}, F_{2}\right]\right)=\int_{\mathbb{T}} \operatorname{Res}_{\delta}\left\{\left[F_{1}, F_{2}\right](I+\mu \delta)^{-1}\right\} \nabla x=\int_{\mathbb{T}}\left(\text { Free }_{\mathcal{E}}\left[F_{1}, F_{2}\right]\right) \frac{\nabla x}{\nu(x)}=0 .
$$

It is enough to check (180) for monomials $F_{1}=A \mathcal{E}^{k}$ and $F_{2}=B \mathcal{E}^{\ell}$. By the use of the property (113) of $\mathcal{E}$ we have

$$
F_{1} F_{2}=A\left(E^{k} B\right) \mathcal{E}^{k+\ell} \quad \text { and } F_{2} F_{1}=B\left(E^{\ell} A\right) .
$$

Therefore Free $_{\mathcal{E}}\left[F_{1}, F_{2}\right]$ is either zero or

$$
\begin{gathered}
\text { Free }_{\mathcal{E}}\left[F_{1}, F_{2}\right]=A\left(E^{k} B\right)-B\left(E^{-k} A\right)=\left(E^{k}-I\right)\left(E^{-k} A\right) B \\
=\left(I-E^{-1}\right)\left(E^{k}+E^{k-1}+\cdots+E\right)\left(E^{-k} A\right) B=\nu(x)[\Phi(A, B)]^{\nabla},
\end{gathered}
$$

where $\Phi(A, B)=\left(E^{k}+E^{k-1}+\cdots+E\right)\left(E^{-k} A\right) B$. Hence

$$
\int_{\mathbb{T}} \text { Free }_{\mathcal{E}}\left[F_{1}, F_{2}\right] \frac{\nabla x}{\nu(x)}=\int_{\mathbb{T}}[\Phi(A, B)]^{\nabla} \nabla x=\Phi(A, B) \mid \begin{aligned}
& x_{x_{*}}^{*} \\
& x_{*}
\end{aligned}
$$

so that (177) is proved for regular-discrete time scales.

(iii) Let $\mathrm{T}$ be a mixed time scale, say, of the form $\mathbb{T}=(-\infty, 0) \cup \mathbb{K}_{q}$, where $(-\infty, 0)$ denotes the real line interval. Then for any $F_{1}, F_{2} \in \Lambda$ we have, taking into account Proposition 26, that 


$$
\operatorname{Tr}\left(\left[F_{1}, F_{2}\right]\right)=\int_{\mathrm{T}} \operatorname{Res}_{\delta}\left\{\left[F_{1}, F_{2}\right](I+\mu \delta)^{-1}\right\} \nabla x=\int_{-\infty}^{0}\left(\operatorname{Res}_{\partial}\left(\left[F_{1}, F_{2}\right]\right) \mathrm{d} x+\int_{\mathbb{K}_{q}}\left(\operatorname{Free}_{\mathcal{E}}\left[F_{1}, F_{2}\right]\right) \frac{\nabla x}{\nu(x)} .\right.
$$

Take for instance $F_{1}=A \delta$ and $F_{2}=B \delta^{-1}$. Then

$$
\begin{aligned}
\operatorname{Res}_{\partial}\left[F_{1}, F_{2}\right] & =\operatorname{Res}_{\partial}\left[A \partial, B \partial^{-1}\right]=A B^{\prime}+A^{\prime} B=(A B)^{\prime}, \text { Free }_{\mathcal{E}}\left[F_{1}, F_{2}\right]=\text { Free }_{\mathcal{E}}\left[\frac{A}{\mu}(\mathcal{E}-I), B(\mathcal{E}-I)^{-1} \mu\right] \\
& =A E(B)-B E^{-1}(A) .
\end{aligned}
$$

Therefore

$$
\begin{aligned}
& \int_{-\infty}^{0}\left(\operatorname{Res}_{\partial}\left[F_{1}, F_{2}\right]\right) \mathrm{d} x=\int_{-\infty}^{0}(A B)^{\prime} \mathrm{d} x=A\left(0^{-}\right) B\left(0^{-}\right), \\
& \int_{\mathrm{K}_{q}} \operatorname{Free}_{\mathcal{E}}\left[F_{1}, F_{2}\right] \frac{\nabla x}{\nu(x)}=\int_{\mathrm{K}_{q}}\left[A E(B)-B E^{-1}(A)\right] \frac{\nabla x}{\nu(x)} \\
& =\sum_{x \in \mathrm{K}_{q}}\left[A(x) B(q x)-A\left(q^{-1} x\right) B(x)\right]=-A\left(0^{+}\right) B\left(0^{+}\right) .
\end{aligned}
$$

Hence

$$
\operatorname{Tr}\left(\left[F_{1}, F_{2}\right]\right)=A\left(0^{-}\right) B\left(0^{-}\right)-A\left(0^{+}\right) B\left(0^{+}\right)=0,
$$

where $A$ and $B$ are $\Delta$-smooth functions on T and hence they are continuous at $x=0$.

Proposition 28: Equation (79) implies that

$$
\frac{d}{\mathrm{~d} t_{n}} L^{k}=\left[A_{n}, L^{k}\right], \quad A_{n}=\left(L^{n / N}\right)_{\geqslant 0},
$$

for all $k=\ell / N$, where $\ell$ is any positive integer.

Propositions 27 and 28 imply the next proposition.

Proposition 29: For all $\ell=0,1, \ldots$ the functionals

$$
H_{\ell}=\operatorname{Tr}\left(L^{\ell / N}\right),
$$

are common constants of motion for the hierarchy (79) and (115).

Note that in proof of Proposition 29 it is, in particular, used the fact that the flows (vector fields) defined by the different members of the hierarchy all commute with each other (see Refs. 2 and 17).

\section{CONCLUSION}

We have developed the Gel'fand-Dikii approach to time scales. So far the integrable systems were studied on $\mathbb{R}, \mathbb{Z}$ or on $\mathbb{K}_{q}$. Here we gave a unified and extended approach. In particular cases when $\mathbb{T}=\mathbb{R}, \mathbb{Z}, \mathbb{K}_{q}$ we find several examples of the integrable systems. We developed the algebra of $\Delta$-pseudo differential and $\mathcal{E}$-shift operators. We established the GD formalism on these algebras and introduced several Lax representations on these algebras. All these Lax representations are straightforward generalizations of the Lax representations on pseudodifferential algebras of integrable systems on $\mathbb{R}$ and the Lax representations of the algebra of shift operators on Z. The Burgers and KdV hierarchies on time scales that we found are the special cases of these Lax representations. We also generalized the Frenkel $\mathrm{KdV}$ hierarchy introduced on $\mathbb{K}_{q}$ to arbitrary 
discrete time scales. We constructed the recursion operators of each example considered in this paper and gave a way of constructing the constants of motions by introducing an appropriate trace form on time scales.

In this work we did not consider the $r$-matrix construction and the Hamiltonian formulation of integrable systems on time scales. The trace form on a general time scale needs a little care. Such a work is in progress and will be communicated in a separate paper.

\section{ACKNOWLEDGMENTS}

This work is partially supported by the Turkish Academy of Sciences and by the Scientific and Technical Research Council of Turkey.

\section{APPENDIX: RECURSION OPERATORS OF FOUR-FIELD SYSTEMS}

We give the recursion operator of the four-field systems on time scale which are studied in example 7.

(1) For the case $k=0, \alpha=-2$, we obtain the recursion relation between the $n+1$ th and $n$th elements of the hierarchy as follows:

$$
\begin{aligned}
w_{n+1}= & w(E+1)^{-1} E\left(v_{n}\right)+E(v)\left(E^{2}-1\right)^{-1} E^{2}\left(w_{n}\right)-v\left(E^{2}-1\right)^{-1} w_{n} \\
& +w(E+1)^{-1}(1-E) w\left(E^{2}-1\right)^{-1} E\left(w_{n}\right)+E^{2}\left(u_{n}\right)+\left(1-E^{2}\right) \eta_{n}, \\
v_{n+1}= & w E\left(u_{n}\right)+v v_{n}-u\left(E^{2}-1\right)^{-1} E^{-1}\left(w_{n}\right)+E(u)\left(E^{2}-1\right)^{-1} E^{2}\left(w_{n}\right) \\
& +\left(1-E^{2}\right) p\left(1-E^{2}\right)^{-1} \frac{p_{n}}{p}+E^{2}\left(p_{n}\right)+\left(E^{-1}(w)-w E\right) \eta_{n}, \\
u_{n+1}= & w E\left(p_{n}\right)+u(E+1)^{-1}\left(E+1+E^{-1}\right) v_{n}-p\left(E^{2}-1\right)^{-1} E^{-2}\left(w_{n}\right) \\
+ & u(E+1)^{-1}(E-1) E^{-1}(w)\left(E^{2}-1\right)^{-1} w_{n}+E(p)\left(E^{2}-1\right)^{-1} E^{2}\left(w_{n}\right) \\
+ & \left(E^{-2}(w)-w E\right) p\left(1-E^{2}\right)^{-1} \frac{p_{n}}{p}+v u_{n}+\left(E^{-1}(v)-v\right) \eta_{n}, \\
p_{n+1}= & u E^{-1}\left(u_{n}\right)+p\left(1+E^{-2}\right) v_{n}+p\left(E^{-1}-E^{-2}\right) w\left(E^{2}-1\right)^{-1} E\left(w_{n}\right) \\
& +\left(E^{-2}(v)-v\right) p\left(1-E^{2}\right)^{-1} \frac{p_{n}}{p}+v p_{n}+\left(E^{-1}(u)-u E^{-1}\right) \eta_{n},
\end{aligned}
$$

where

$$
\eta_{n}=\left(E^{2}(p)-E(p) E^{2}\right)^{-1}\left[E^{2}(u) E\left(p_{n}\right)+E^{2}(p) u_{n}+\left(u E^{2}(p)-E^{2}(u) E(p) E^{-1}\right)\left(1-E^{2}\right)^{-1} E^{2}\left(\frac{p_{n}}{p}\right)\right] .
$$

(2) For the case $k=1, \alpha=-2$, the recursion relation between the $n+1$ th and $n$th elements of the hierarchy is given by

$$
\begin{gathered}
\bar{u}_{n+1}=E^{-2}\left(\bar{w}_{n}\right)+\bar{u}(1+E)^{-1}(E-1) \bar{u}\left(1-E^{2}\right)^{-1} E\left(\bar{u}_{n}\right)+\bar{v} \bar{u}_{n}+\bar{u}(1+E)^{-1} \bar{v}_{n} \\
+\left(E^{-1}(\bar{v})-\bar{v}\right)\left(1-E^{2}\right)^{-1} \bar{u}_{n}+\left(1-E^{-2}\right) \bar{\zeta}_{n} \\
\bar{w}_{n+1}=\bar{u} E^{-1}\left(\bar{q}_{n}\right)+\bar{w}(1+E)^{-1}\left(E^{2}+E+1\right) \bar{v}_{n}+\left(E^{-1}(\bar{q})-\bar{q} E^{4}\right)\left(1-E^{2}\right)^{-1} \bar{u}_{n}+\bar{w}(1+E)^{-1}(1-E) E(\bar{u}) \\
\times\left(1-E^{2}\right)^{-1} E^{2}\left(\bar{u}_{n}\right)+\bar{v} \bar{w}_{n}+(E(\bar{v})-\bar{v}) \bar{\zeta}_{n}+\left(E^{2}(\bar{u})-\bar{u} E^{-1}\right) \bar{q}\left(E^{2}-1\right)^{-1} E^{2}\left(\frac{\bar{q}_{n}}{\bar{q}}\right),
\end{gathered}
$$




$$
\begin{aligned}
\bar{q}_{n+1}= & \bar{v} \bar{q}_{n}+\bar{w} E\left(\bar{w}_{n}\right)+\bar{q}\left(1+E^{2}\right) \bar{v}_{n}+\left(E^{2}(\bar{v})-\bar{v}\right) \bar{q}\left(E^{2}-1\right)^{-1} E^{2}\left(\frac{\bar{q}_{n}}{\bar{q}}\right) \\
& +\bar{q}(1+E)^{-1}\left(1-E^{2}\right) E(\bar{u})\left(1-E^{2}\right)^{-1} E^{2}\left(\bar{u}_{n}\right)+(E(\bar{w})-\bar{w} E) \bar{\zeta}_{n}, \\
\bar{v}_{n+1}= & E^{-2}\left(\bar{q}_{n}\right)+\bar{u} E^{-1}\left(\bar{w}_{n}\right)+\left(E^{-1}(\bar{w})-\bar{w} E^{3}\right)\left(1-E^{2}\right)^{-1} \bar{u}_{n}+\bar{v} \bar{v}_{n}+\left(E(\bar{u})-\bar{u} E^{-1}\right) \bar{\zeta}_{n} \\
& +\left(1-E^{-2}\right) \bar{q}\left(E^{2}-1\right)^{-1} E^{2}\left(\frac{\bar{q}_{n}}{\bar{q}}\right),
\end{aligned}
$$

where

$$
\bar{\zeta}_{n}=\left(\bar{q} E^{2}-E(\bar{q})\right)^{-1}\left[\bar{q} E^{2}\left(\bar{w}_{n}\right)+\left(E^{2}(\bar{w}) \bar{q} E-\bar{w} E(\bar{q})\right)\left(E^{2}-1\right)^{-1} E\left(\frac{\bar{q}_{n}}{\bar{q}}\right)\right] .
$$

From the recursion relations obtained in both cases, we can construct the recursion operators of the hierarchies.

${ }^{1}$ A. S. Fokas and I. M. Gel'fand, "Bi-Hamiltonian structures and integrability," in Recent Developments in Soliton Theory, edited by A. S. Fokas and V. E. Zakharov (Springer-Verlag, New York, 1992).

${ }^{2}$ L. A. Dickey, Soliton Equations and Hamiltonian Systems (World Scientific, Singapore, 1991).

${ }^{3}$ M. Blaszak, Multi-Hamiltonian Theory of Dynamical Systems (Texts and Monographs in Physics) (Springer, Berlin, 1998).

${ }^{4}$ E. Frenkel, Int. Math. Res. Notices 1996, 55 (1996).

${ }^{5}$ B. Khesin, V. Lyubashenko, and C. Roger, J. Funct. Anal. 143, 55 (1997).

${ }^{6}$ M. Adler, E. Horozov, and P. van Moerbeke, Phys. Lett. A 242, 139 (1998).

${ }^{7}$ B. Aulbach and S. Hilger, "Linear dynamic process with inhomogeneous time scale," Nonlinear Dynamics and Quantum Dynamical Systems (Gaussing, 1990), Math. Res. 59 (Akademie Verlag, Berlin, 1990), pp. 9-20.

${ }^{8}$ S. Hilger, Results Math. 18, 18 (1990).

${ }^{9}$ V. Kac and P. Cheung, Quantum Calculus (Springer-Verlag, New York, Berlin, Heidelberg, 2002).

${ }^{10}$ M. Bohner and A. Peterson, Dynamic Equations on Time Scales: An Introduction with Applications (Birkhauser, Boston, 2001).

${ }^{11}$ Advances in Dynamic Equations on Time Scales, edited by M. Bohner and A. Peterson (Birkhauser, Boston, 2003).

${ }^{12}$ F. M. Atıcı and G. Sh. Guseinov, J. Comput. Appl. Math. 141, 75 (2002).

${ }^{13}$ G. Sh. Guseinov, J. Math. Anal. Appl. 285, 107 (2003).

${ }^{14}$ M. Gürses, A. Karasu, and V. V. Sokolov, J. Math. Phys. 40, 6473 (1999).

${ }^{15}$ M. Blaszak, Rep. Math. Phys. 48, 27-38 (2001).

${ }^{16}$ M. Gürses and K. Zheltukhin, J. Math. Phys. 42, 1309 (2001).

${ }^{17}$ G. Wilson, Math. Proc. Cambridge Philos. Soc. 86, 131 (1979). 